\title{
Hybrid add-drop filter based on one-dimensional photonic crystal Fabry-Perot resonator
}

\author{
Ido E. Dotan, Damian Goldring, and David Mendlovic \\ Faculty of Engineering, Tel-Aviv University, Tel-Aviv 69978, Israel \\ ido.dotan@gmail.com
}

\begin{abstract}
We devised an approach for realizing add-drop filters. This approach uses strip waveguides to couple light into photonic crystal based resonators. We used silicon on insulator substrate with a $240 \mathrm{~nm}$ thick Si layer. Theoretical analysis, simulation and experiments were conducted. We compared the new device to a ring-based add-drop filter and found that for rings with radii smaller than $1.414 \mu \mathrm{m}$, our device has significant advantages.
\end{abstract}

Keywords: Photonic crystals, resonators, filters.

\section{INTRODUCTION}

The technology of integrated optics has been attracting a lot of attention for the past three decades, and is rapidly developing, mainly as a result of technological advances in the semiconductor industry. This technology is applicable in optical communication systems and can be realized in silicon photonics for lasers, modulators, switches and filters [1-7]. One important application is the add-drop filter (ADF) [8-12] which is a device transferring data between two buses through the use of a resonator. The most common way for realizing ADF is by using a ring resonator. However in cases where a high free spectral range (FSR) is needed or smaller device required, the ring resonator might not meet the design requirements, and thus, other realizations should be considered. Recent years developments enabled the realization and fabrication of photonic crystals $(\mathrm{PhC})$ structures. An ADF realization using 2D $\mathrm{PhC}$ enables the fabrication of high quality (Q) factor in small dimensions; however it raises the problem of bandgap matching between $\mathrm{PhC}$ waveguides and $\mathrm{PhC}$ cavity, and mode matching between $\mathrm{PhC}$ waveguide and strip waveguide.

In this letter we suggest and demonstrate a realization of an $\mathrm{ADF}$ based on a $\mathrm{PhC}$ resonator. We use two 1D PhC structures as partial mirrors to form a Fabry-Perot (FP) resonator. Unlike in previous realizations of $1 \mathrm{D} \mathrm{PhC}$ based $\mathrm{FP}$ where the device was embedded into the data bus, we suggest the use of the device to couple two data buses and achieve an ADF configuration. The coupling of light into and out-of the resonator is done using curved standard rectangular (strip) waveguides. Although PhC-based ADFs have been demonstrated before, we present here an approach that will significantly reduce the design hurdles introduced in former devices. This approach utilizes both $\mathrm{PhC}$ based structures and regular (non periodic) structures. The latter enables us to use the advantages of $\mathrm{PhCs}$ while avoiding some of their problems, such as, coupling to $\mathrm{PhC}$ structures and spectral line width restrictions of $\mathrm{PhC}$ waveguides.

\section{ADF BASED ON 1D PHC FABRY-PEROT RESONATOR}

We turn to analyze our hybrid ADF design, shown in Fig. 1. The ADF consists of two data busses in a standard strip configuration (Fig. 1(a)). The coupling between the busses is resonant-assisted using the FP resonator shown in detail in Fig. 1(b). The FP resonator consists of two partial mirrors (reflectors), each realized by a 1D PhC. The PhC is realized by holes etched into a waveguide of width $\mathrm{W}=470 \mathrm{~nm}$ causing a refractive index modulation $[13,14]$. The two sets of holes include tapering of the $\mathrm{PhC}$ radii to obtain improved mode 
matching hence higher reflectivity [15], and are separated with distance L as marked in Fig. 1(a). Highly refractive mirrors can be fabricated with PhCs [2, 15-17], creating a strong confinement of light inside the resonator, yielding high quality factors [15].

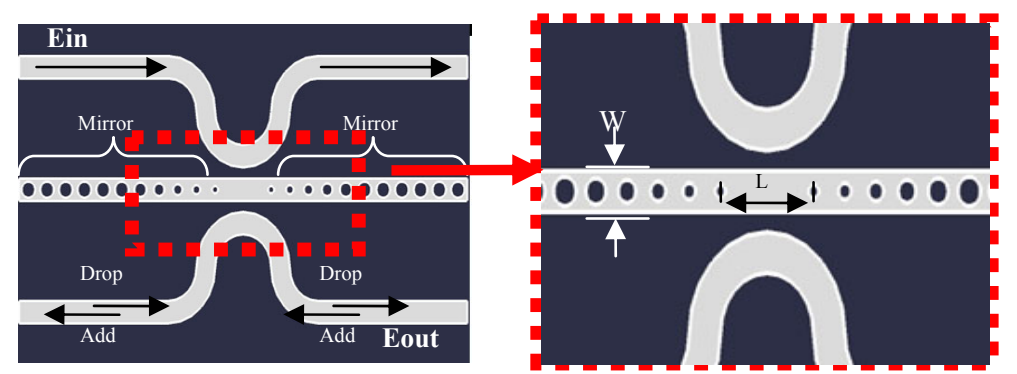

(a)

(b)

Fig. 1. (a) Schematic description of hybrid ADF. (b) Zooming in on the resonator area, realized with $1 \mathrm{D} \mathrm{PhC} \mathrm{mirrors.}$

In order to be able to predict the output of our device, we need to mathematically analyze the energy transfer between the input bus and the right "add/drop" bus, as a function of the wavelength. Let us assume that light signal is fed into the device through the input bus. Part of that light will be coupled to the FP resonator according to the field coupling coefficient $\kappa_{c}$. Similarly, light propagating inside the resonator (in a specific direction) will be coupled out to both data busses and to both directions. In the first encounter of the light with the coupling area, some of it will be coupled to the resonator, and a small portion will be coupled out to the second bus but experience a weaker coupling coefficient $\kappa_{\mathrm{c}} / \mathrm{a}$ where $\mathrm{a}>1$. We denote by $\mathrm{R}_{\mathrm{m}}=\left|\mathrm{r}_{\mathrm{m}}\right|^{2}$ the PhC mirror's reflectivity for intensity and field, respectively, and the propagation constant of the wave in the resonator by $\mathrm{k}=2 \pi \mathrm{n} / \lambda$ ( $n$ is the effective index of the optical mode in the resonator). Using FP formulation, we obtain the field in the drop bus as function of an excitation field in the input bus:

$$
E_{\text {out }}=E_{\text {in }}\left(j \kappa_{c}\right)^{2}\left(\frac{1}{a}+\left[\frac{\sqrt{1-\left(\frac{\kappa_{c}}{a}\right)^{2}} \sqrt{1-2 \kappa_{c}^{2}} R_{m} e^{j 4 k l}}{1-\left(1-2 \kappa_{c}^{2}\right) R_{m} e^{j 4 k l}}\right]\right)
$$

In order to be able to use Eq. (1), we need to have some knowledge the reflectivity of the $\mathrm{PhC}$ mirrors and about the coupling coefficient, as a function of the wavelength. We used a commercial (Rsoft ${ }^{\mathrm{TM}}$ ) simulation tool to find these parameters. The results for the busresonator transmittance coefficient $\mathrm{T}=\sqrt{1-\kappa^{2}}$ and the reflectance of the $1 \mathrm{D}$ tapered $\mathrm{PhC}$ mirror are shown in Fig. 2(a). These results were then used, together with Eq. (1), to simulate the spectral response at the output, as shown in Fig. 2(b) as the blue line. We assumed that the distance between the two mirrors is $\mathrm{L}=45 \mu \mathrm{m}$. 


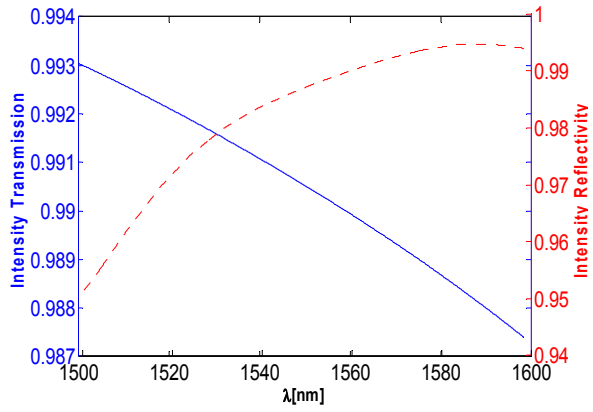

(a)

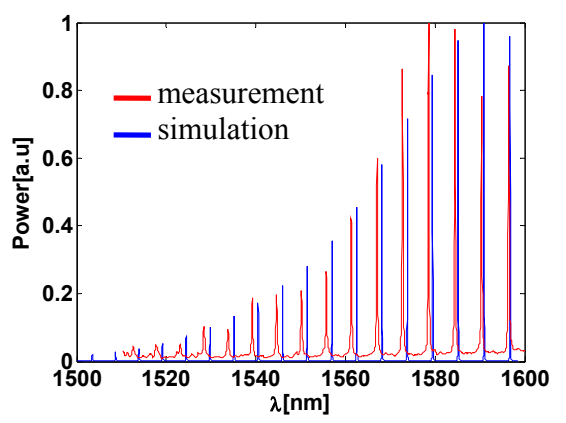

(b)

Fig. 2. (a) Simulation results of bus to resonator power coupling coefficient (blue) and Reflectivity of tapered mirror with period $=403 \mathrm{~nm}$ (dashed red line). (b) "Drop" bus output of an ADF with tapered mirrors with period $=403 \mathrm{~nm}$ measured (red line), simulated (blue line).

This calculation was done only for the right hand of the add/drop bus, but all other three ports get similar outputs. This suggests that the relevant output port receives only $25 \%$ of the intensity as in the $2 \mathrm{D} \mathrm{PhC}$ case. We can overcome this problem by designing $\mathrm{PhC}$ mirrors for the other two outputs; however, we will lose the ability to use this device as an ADF rather than a regular filter.

\section{EXPERIMENTAL RESULTS}

We have fabricated the device on a Silicon on Insulator (SOI) substrate $(250 \mathrm{~nm}$ thick $\mathrm{Si}$ device layer on top of a $3 \mu \mathrm{m}$ thick $\mathrm{SiO}_{2}$ layer), using electron beam lithography followed by the reactive ion etching (RIE) process. Finally, we used a $1 \mu \mathrm{m}$ thick $\mathrm{SiO}_{2}$ as a cover layer. Fig. 3(a) shows high-resolution scanning electron microscope (HRSEM) images of the fabricated device and Fig. 3(b) shows 1D PhC reflector.

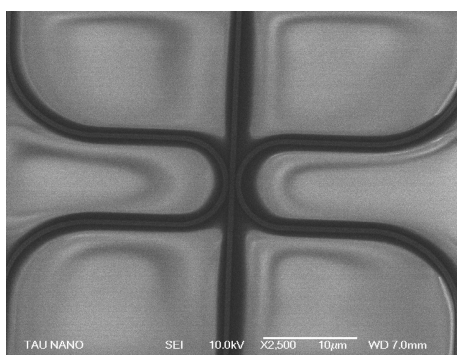

(a)

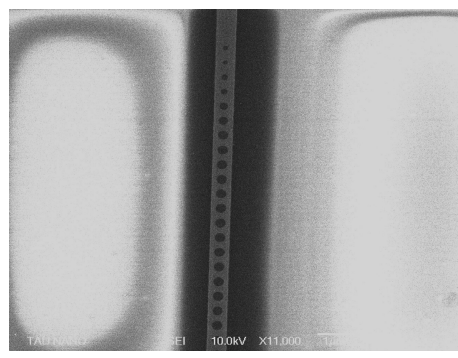

(b)

Fig. 3. High-resolution SEM images of the add-drop PhC based Fabry-Perot resonator. (a) Coupling area, (b) PhC tapered mirror.

For the tapering of the $\mathrm{PhC}$ reflector we used the following parameters: Main radius is $115 \mathrm{~nm}$, smallest radius in taper is $25 \mathrm{~nm}$, number of main holes is 19, and the number of (linear) tapered holes equals 9. The distance between mirrors is $\mathrm{L}=45 \mu \mathrm{m}$, or the measurements we used tapered fibers to couple the field $E_{\text {in }}$ into the device (Fig. 1(a)) to measure $E_{\text {out }}$ at the "drop" bus. The measurements results for a device with mirror's period $403 \mathrm{~nm}$ are shown in (red line), along the simulation results (blue line). 
The slight differences between simulation and measurement are the result of the fact that the simulation did not account for imperfections in the manufacturing causing edge roughness of the waveguide, and uneven holes in the mirror. Despite all that, the simulation is in good agreement with the experimental results.

\section{DISCUSSION}

In order for the idea to be complete, we need to compare the suggested design to the common ADF structure, i.e., the ring resonator. We compared the two configurations in terms of the optical losses in the resonators which are the limiting factor for reducing the ring's radius below a certain limit. The loss mechanism in the FP resonator is the reflection loss of the mirrors, while in the ring resonator, the losses are caused by the curvature of the waveguide itself. In order to isolate these loss mechanisms from the propagation loss in a regular ridge waveguide (which is common for both designs), we compared designs that have the same optical roundtrip length in the resonator. We shall begin this discussion with a short review on the ring resonator structure and operation basics. The ring resonator is a strip waveguide curved with radius $\mathrm{R}$ and width $\mathrm{W}=\mathrm{R}_{2}-\mathrm{R}_{1}$, as shown in Fig. 4. Light propagating along the ring will suffer destructive interference unless the accumulated phase is a multiple of $2 \pi$ for each roundtrip, in which case the device is said to be on resonance.

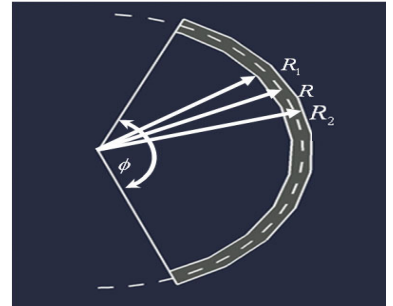

(a)

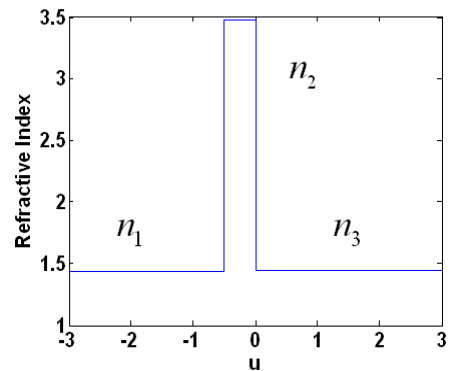

(b)

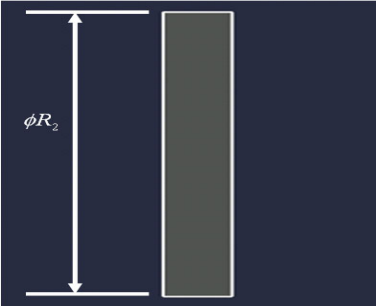

(c)

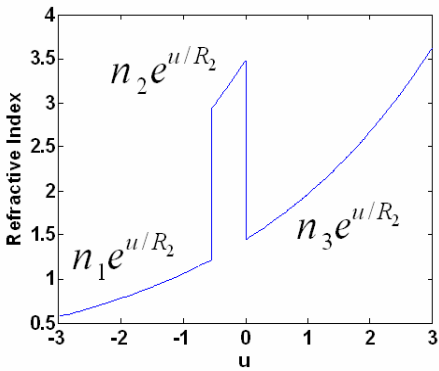

(d)

Fig. 4. Transformation of a curved waveguide to a straight waveguide. (a) Curved waveguide with radius $\mathrm{R}$, and (b) its refractive index along the radial cross section. (c) Straight waveguide transformed from (a), and (d) its refractive index along the cross-section.

The performances of such a ring depend on the refractive index difference between waveguide and cladding, radius of curvature and the dimensions of the waveguide. The curvature itself causes loss in the power of the propagating light. In order to calculate these losses we used a conformal transformation that transforms the ridged profile ring (in the radial direction) into an exponential profile of a straight waveguide, as elaborately described in Refs. 18-22 and as shown in Fig. 4.

Using the beam propagation method (BPM) for the transformed waveguide, we simulated the curved rings with different radii. The propagation loses were fitted to the exponential 
function $\mathrm{I}(\mathrm{L})=\mathrm{I}_{0} \exp \left(-\alpha_{R} \mathrm{~L}\right)$, where $\alpha_{R}$ is losses per unit length. These losses can be compared to the losses in the FP $\alpha_{\mathrm{FP}}=1 / 2 \mathrm{D} \cdot \ln \left(1 / \mathrm{r}_{\mathrm{FP}}{ }^{2}\right)$, where $\mathrm{r}_{\mathrm{FP}}$ is the intensity reflectivity of a mirror and $\mathrm{D}$ is the distance between inner holes of both mirror. Figure 5(a) shows the losses as a function of the ring radius, compared to a loss graph (red line) of a FP resonator assuming an intensity reflectivity $\mathrm{r}_{\mathrm{FP}}=0.997$ and length equals to half of the ring circumference.

The critical ring radius, under which the FP resonator with reflectivity $r_{\mathrm{FP}}=0.997$ is preferred, is $1.414 \mu \mathrm{m}$. Radii smaller than the critical radius will result in high losses in the ring structure, causing shorter photon lifetime inside the resonator, and hence smaller $\mathrm{Q}$ factor. Notice that the losses in the ring increase rapidly as the radius becomes smaller than $1.414 \mu \mathrm{m}$, whereas in the FP case, the loss change is milder. Figure 5(b) shows the dependence of the minimum radius required for a ring to be more efficient than FP, as a function of the FP mirror reflectivity. Intuitively, as the reflectivity value increases, the more efficient the FP resonator becomes, hence a larger ring radius is required to compete with the FP. Better fabrication technology enables the FP to be a real competition to the ring resonator, especially when used in the hybrid configuration we described.

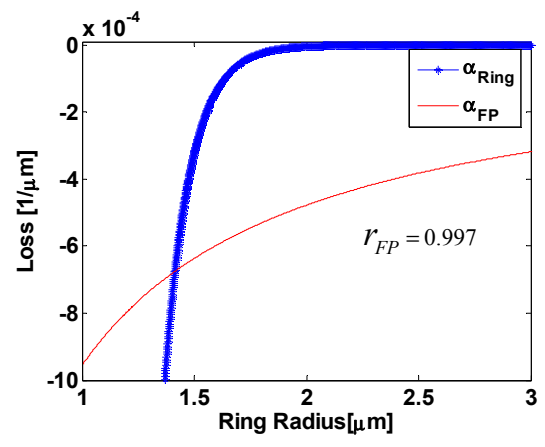

(a)

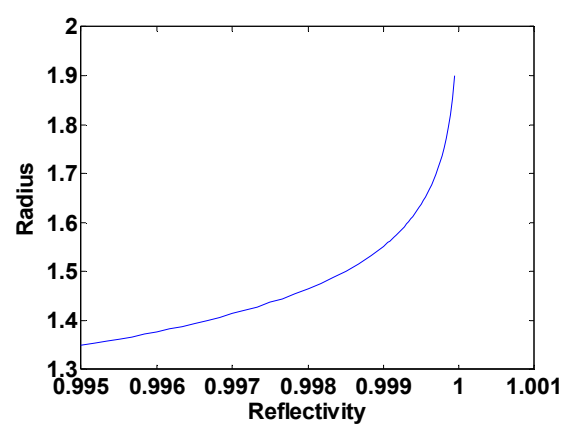

(b)

Fig. 5. (a) Comparison between the losses inside Ring resonator (stared), and losses caused by Fabry-Perot mirrors (continuous line). (b) Minimum radius required for a ring resonator to compete with FP resonator with different mirror reflectivity.

\section{References}

[1] T. Tanabe, M. Notomi, S. Mitsugi, A. Shinya, and E. Kuramochi, "All-optical switches on a silicon chip realized using photonic crystal nanocavities," Appl. Phys. Lett. 87, 151112-151113 (2005) [doi: 10.1063/1.2089185].

[2] B. Schmidt, Q. Xu, J. Shakya, S. Manipatruni, and M. Lipson, "Compact electro-optic modulator on silicon-on-insulator substrates using cavities with ultra-small modal volumes," Opt. Exp. 15, 40-42 (2007) [doi:10.1364/OE.15.003140].

[3] Y. Akahane, T. Asano, B.-S. Song, and S. Noda, "High-Q photonic nanocavity in a two-dimensional photonic crystal," Nature 425, 944-947 (2003) [doi:10.1038/nature02063].

[4] J. S. Li, "Terahertz modulator using photonic crystals," Opt. Commun. 269, 98-101 (2007) [doi:10.1016/j.optcom.2006.07.053].

[5] J. Li, J. He, and Z. Hong, "Terahertz wave switch based on silicon photonic crystals," Appl. Opt. 46, 5034-5037 (2007) [doi:10.1364/AO.46.005034]. 
[6] C. Vazquez, S. Vargas, J. M. S. Pena, and P. Corredera, "Tunable optical filters using compound ring resonators for DWDM," IEEE Photonics Technol. Lett. 15, 1085-1087 (2003) [doi:10.1109/LPT.2003.815328].

[7] E. Waks and J. Vuckovic, "Ultrahigh-Q photonic crystal nanocavities realized by the local width modulation of a line defect," Opt. Exp. 13, 5064-5073 (2005) [doi:10.1364/OPEX.13.005064].

[8] S. Fan, P. Villeneuve, J. Joannopoulos, and H. Haus, "Channel drop filters in photonic crystals," Opt. Exp. 3, 4-11 (1998).

[9] H. Takano, B.-S. Song, T. Asano, and S. Noda, "Highly efficient multi-channel drop filter in a two-dimensional hetero photonic crystal," Opt. Exp. 14, 3491-3496 (2006) [doi:10.1364/OE.14.003491].

[10] O. Schwelb, "Transmission, group delay, and dispersion in single-ring optical resonators and add/drop filters--A tutorial overview," J. Lightwave Technol. 22, (2004) [doi:10.1109/JLT.2004.827666].

[11] Y. Akahane, T. Asano, H. Takano, B.-S. Song, Y. Takana, and S. Noda, "Twodimensional photonic-crystal-slab channel drop filter with flat-top response," Opt. Exp. 13, 2513-2530 (2005) [doi:10.1364/OPEX.13.002512].

[12] M. Notomi, A. Shinya, S. Mitsugi, E. Kuramochi, and H.-Y. Ryu, "Waveguides, resonators and their coupled elements in photonic crystal slabs," Opt. Exp. 12, 1551 (2004) [doi:10.1364/OPEX.12.001551].

[13] S. G. Johnson and J. D. Joannopoulos, Photonic Crystals--The Road from Theory to Practice, Kluwer, Boston (2002).

[14] J. D. Joannopoulos, P. R. Villeneuve, and S. Fan, "Photonic crystals: Putting a new twist on light," Nature 386, 143-149 (1997) [doi:10.1038/386143a0].

[15] A. R. M. Zain, N. P. Johnson, M. Sorel, and R. M. D. L. Rue, "Ultra high quality factor one dimensional photonic crystal photonic wire micro-cavities in silicon-on-insulator," Opt. Exp. 16, 12084-12089 2008 [doi:10.1364/OE.16.012084].

[16] D. Goldring, U. Levy, I. E. Dotan, A. Tsukernik, M. Oksman, I. Rubin, Y. David, and D. Mendlovic, "Experimental measurement of quality factor enhancement using slow light modes in one dimensional photonic crystal," Opt. Exp. 16, 5585-5595 (2008) [doi:10.1364/OE.16.005585].

[17] D. Goldring and D. Mendlovic, "New design method for one-dimensional photonic crystal reflectors in strip waveguides," IEEE 24th Conv. Electrical Electron. Engineer. Israel, 115-118 (2006) [doi: 10.1109/EEEI.2006.321114].

[18] M. Heiblum and J. Harris, "Analysis of curved optical waveguides by conformal transformation," IEEE J. Quantum Electron. 11, 75-83 (1975) [doi:10.1109/JQE.1975.1068563].

[19] S. C. M. Lidgate, P. Sewell, and T. M. Benson, "Conformal mapping: Limitations for waveguide bend analysis," IEEE Proc. Sci. Measure. Technol. 149, 262-266 (2002) [doi: 10.1049/ip-smt:20020597].

[20] M. K. Chin and S. T. Ho, "Design and modeling of waveguide-coupled single-mode microring resonators," J. Lightwave Technol. 16, 1433-1446 (1998) [doi:10.1109/50.704609].

[21] A. Melloni, F. Carniel, R. Costa, and M. Martinelli, "Determination of bend mode characteristics in dielectric waveguides," J. Lightwave Technol. 19, 571-577 (2001) [doi:10.1109/50.920856].

[22] R. Baets and P. E. Lagasse, "Loss calculation and design of arbitrarily curved integrated-optic waveguides," $J$. Opt. Soc. Am. 73, $177-182 \quad$ (1983) [doi:10.1364/JOSA.73.000177]. 